\title{
Incremental Growth Lines in Mouse Molar Dentin Represent 8-hr Ultradian Rhythm
}

\author{
Ryutaro Ono $^{1,2, *}$, Nobuya Koike ${ }^{1, *}$, Hitoshi Inokawa1, Yoshiki Tsuchiya ${ }^{1}$, \\ Yasuhiro Umemura ${ }^{1}$, Toshiro Yamamoto ${ }^{2}$, Narisato Kanamura ${ }^{2}$ and Kazuhiro Yagita ${ }^{1}$ \\ ${ }^{1}$ Department of Physiology and Systems Bioscience, Graduate School of Medicine, Kyoto Prefectural University of Medicine \\ and ${ }^{2}$ Department of Dental Medicine, Graduate School of Medicine, Kyoto Prefectural University of Medicine, Kawaramachi- \\ Hirokoji, Kamigyo-ku, Kyoto 602-8566, Japan
}

Received June 5, 2019; accepted November 5, 2019; published online December 20, 2019

\begin{abstract}
Rhythmic incremental growth lines occur in dental hard tissues of vertebrates, and dentinogenesis in rodent incisors is suggested to be controlled by the 24-hr circadian clock. Rodent incisors continue to grow throughout the animal's life; however, similar to human teeth, rodent molars stop growing after crown formation. This similarity suggests that the mouse molar is an excellent model to understand the molecular mechanisms underlying growth of human teeth. However, not much is known about the rhythmic dentinogenesis in mouse molars. Here, we investigated the incremental growth lines in mouse molar dentin using tetracycline as the growth marker. The incremental growth lines were observed to be generated at approximately 8-hr intervals in wild-type mice housed under 12:12 hr light-dark conditions. Moreover, the 8-hr rhythmic increments persisted in the wild-type and Bmal1 $1^{-/-}$ mice housed in constant darkness, where $\mathrm{Bmal1}^{-/-}$mice become behaviorally arrhythmic. These results revealed that the dentinogenesis in mouse molars underlie the ultradian rhythms with around 8-hr periodicity. Further, the circadian clock does not seem to be involved in this process, providing new insight into the mechanisms involved in the tooth growth.
\end{abstract}

Key words: dentinogenesis, biological rhythms, circadian clocks, tetracycline, tooth

\section{Introduction}

Rhythmic banding patterns are commonly observed throughout the biological organisms living on the earth. Examples include the annual growth rings in trees, color of butterflies' wings, and Haversian canals in bones [15]. In human dentin sections, alternating dark- and light-colored layers were discovered more than 100 years ago by Owen, Andresen, and Von Ebner [1, 22, 28]. These rhythmic pat-

\footnotetext{
* These authors equally contributed to this work.

Correspondence to: Ryutaro Ono, M.D., Department of Physiology and Systems Bioscience, Graduate School of Medicine, Kyoto Prefectural University of Medicine and Department of Dental Medicine, Graduate School of Medicine, Kyoto Prefectural University of Medicine, Kawaramachi-Hirokoji, Kamigyo-ku, Kyoto 602-8566, Japan.

E-mail: rono0718@koto.kpu-m.ac.jp
}

terns, called incremental growth lines, have been observed in several vertebrates, including rodents $[18,24]$, rabbits [21], non-human primates [5], and alligators [16]. Dentin is biological composites containing hydroxyapatite crystals and organic type-I collagens, and forms the major portion of a tooth by weight and volume [10]. Dentinogenesis begins from the dentin-enamel junction (DEJ) and proceeds inward to the dental pulp. During dentinogenesis, odontoblasts produce extracellular matrix proteins, including typeI collagens and dentin sialoproteins, deposition of which facilitates the mineralization of the dentin matrix $[10,30]$. Dentin is formed incrementally, suggesting the involvement of circadian clock mechanisms in dentinogenesis.

The circadian clock controls many behavioral and physiological processes with a periodicity of approximately 24-hr, enhancing the efficiency and survival of organisms by enabling them to anticipate and adapt to changing envi- 
ronmental conditions. The master pacemaker is situated in the suprachiasmatic nucleus $(\mathrm{SCN})$ of the hypothalamus and temporally coordinates peripheral oscillators in various tissues and cells throughout the body $[32,34]$. The circadian molecular oscillators comprise transcriptional/translational feedback loops of clock genes and can be monitored even in cultured cells $[2,7,31]$. CLOCK and BMAL1, transcriptional activators in mammals, heterodimerize and induce Per (Per1, 2, 3) and Cry $(C r y 1,2)$ transcription. PER and CRY proteins translocate into the nucleus and associate with the CLOCK/BMAL1 heterodimer to form a complex that represses their expressions [26]. In rabbit incisors, the incremental growth lines in dentin were reportedly formed at daily intervals [21]. Circadian rhythms have also been demonstrated in the collagen-synthetic and -secretory activities of odontoblasts in rat incisors by using $3 \mathrm{H}$-proline tracers that label collagens in dentinogenesis. Ohtsuka et al. [19] found that twice as much collagen is secreted during the 12-hr daytime as during the 12-hr nighttime. SCN is responsible for the generation of circadian rhythm that results in the production of daily incremental growth lines in the dentin of rat incisors, and fully- or partially-lesioned SCN can lead to permanent or temporary loss of circadian dentin increments [20]. Taken together, these studies suggest that dentinogenesis in rodent incisors is controlled by circadian clock mechanisms.

Rodents have two kinds of teeth, incisors and molars, differing in shape, function, position, and growth characteristics. Incisors have open roots allowing continuous growth throughout the rodent's lifespan. As a result, the nearly equal rates of tooth wear and eruption maintain a constant tooth length [29]. In contrast, rodent molars stop growing after crown formation, similar to human teeth [4, 13]. Therefore, the mouse molar can be considered to be an excellent model to investigate the molecular mechanisms underlying growth of human teeth. However, to date, no studies have focused on rhythmic dentinogenesis in mouse molars. In the present study, we have analyzed the incremental growth lines in mouse molar dentin and determined the periodicity by chronological labeling, using tetracycline as a growth marker. We observed 8-hr ultradian dentin increments in mouse upper second molars and concluded that the circadian clock is not involved in the generation of these rhythmic patterns.

\section{Materials and Methods}

\section{Animals and breeding conditions}

Wild-type and homozygous $\mathrm{Bmall}^{-/-}$mice were obtained by mating heterozygous pairs [3]. They were divided into two groups immediately after birth and housed in light-tight boxes under normal 12-hr light and 12-hr dark cycle (12:12 LD: lights on at 8:00 AM, lights off at 8:00 PM) or under constant darkness (DD), with ad libitum access to food and water. At 1 week of age, mice were injected intraperitoneally with tetracycline $(30 \mathrm{mg} / \mathrm{kg})$ at
2:00 PM as described in Ohno [17], and the injections were repeated every 7 days for 8 weeks. For the mice housed in $\mathrm{DD}$, cage cleaning and tetracycline injections were carried out in the darkness by investigators wearing night-vision goggles (Armasight Spark-G, FLIR systems, Wilsonville, OR, US). For genotyping, they were exposed to light for 30 min at 2-3 weeks of age. At 14 days after the last injection, the 10-week-old mice were sacrificed to prepare the teeth sections. Molars, including the maxillary bones, were removed and divided into the right and left sides. The segments were then prepared for histological analysis. All animal experiments were performed in accordance with the guidelines of the Kyoto Prefectural University of Medicine Animal Care and Use Committee.

\section{Decalcified sections}

The right-side samples were immersed immediately in $10 \%$ buffer formalin and the decalcified samples were prepared and sectioned by New Histo. Science Laboratory (Tokyo, Japan). Briefly, the specimens were decalcified with $10 \%$ EDTA/10 mM Tris-HCl $(\mathrm{pH} \mathrm{7.2)}$ for about 2 weeks, dehydrated in a graded series of ethanol and xylene, and then embedded in paraffin. The paraffin-embedded samples were sectioned to $5-\mu \mathrm{m}$ thickness using a cryostat (RM2245, Leica Biosystems, Wetzlar, Germany). The upper second molar, including the jawbone, was sliced longitudinally along the tooth axis and approximately midway between the buccal and palatal surfaces. All sections were mounted on glass slides and stained with Carazzi's hematoxylin and eosin after rinsing with running water. Finally, a cover slip was applied to the slide.

\section{Frozen sections}

Frozen sections were prepared by Kureha Special Laboratory (Fukushima, Japan). Following the methods described previously [9], the left sides were cut into an appropriate size and dipped in a $10 \%-30 \%$ sucrose solution $\left(4^{\circ} \mathrm{C}\right)$ for about $60 \mathrm{~min}$. The samples were immersed in $4 \%$ carboxymethyl cellulose (CMC) gel and dipped in hexane $\left(-94^{\circ} \mathrm{C}\right)$ with a cooling device. The frozen CMC blocks were attached to the cryomicrotome (CM3050S, Leica Biosystems, Wetzlar, Germany). The surface was then covered with an adhesive-coated film (Cryofilm type $2 c(9)$, Section-lab, Hiroshima, Japan) and sliced into 7- $\mu \mathrm{m}$ thick sections. The sections were placed on a glass slide and coated with encapsulant (SCMM-R2, Section-lab, Hiroshima, Japan).

\section{Measurements and chronological analysis}

Decalcified teeth sections were observed and photographed using a light microscope (Eclipse E800, Nikon, Tokyo, Japan) to measure the intervals of the incremental growth lines, and densitometry of dentin increments was conducted with the image analyzer (imageJ, National Institutes of Health, Bethesda, Maryland, U.S.) at $0.12 \mu \mathrm{m}$ intervals in the direction of an arrow. Following a previ- 
ously described method [5], four or five consecutive lines in the coronal dentin of the upper second molar were marked perpendicular to the plane of the lines, and the distance between the first and last marks was measured with an ocular micrometer mounted on the light microscope. This measured distance was then divided by the number of spaces to determine the average interval of the adjacent incremental growth lines $(\mu \mathrm{m})$.

Tetracycline-labeled bands in frozen sections were observed using a fluorescence microscope (BZ-X710, Keyence, Osaka, Japan) with a GFP filter (OP-87763, Keyence, Osaka, Japan). The distance between the first and second successive fluorescent bands from DEJ in coronal dentin of the upper second molar was determined as the weekly dentin apposition rate and divided by 7 to calculate the daily growth. The periodicity of incremental growth lines (in hr) was calculated using the following equation,

$$
\text { Periodicity (hr) }=24 \times \frac{7 \times \mathrm{IL}(\mu \mathrm{m})}{\mathrm{TB}(\mu \mathrm{m})}
$$

where IL is the interval of the incremental growth lines and TB is the interval of the tetracycline-labeled bands. Statistical differences were evaluated using one-way ANOVA and were calculated using Microsoft Excel. All data were given as mean $\pm \mathrm{SD}$.

\section{Results}

\section{Incremental growth lines in mouse molar dentin represented} approximately 8-hr rhythms

We first examined the decalcified molar dentin sections from mice raised under 12:12 LD conditions. The incremental growth lines were clearly observed as darkand light-colored layers and tended to run parallel with the dentin surface (Fig. 1A). These gradually disappeared on the inner side near the pulp cavity. We measured the intervals of incremental growth lines in 30-100 $\mu \mathrm{m}$ inward from DEJ (roughly one-third of the whole dentin) in the section where the lines were most readily apparent and found an average interval of $3.36 \pm 0.09 \mu \mathrm{m}$ (Table 1).

To analyze the periodicity of incremental growth lines, we next measured the intervals of tetracycline-labeled bands in frozen sections. The series of accentuated labels were clearly observed in the fluorescent microscopic images of frozen dentin sections (Fig. 1C). To quantify dentin growth in the area corresponding to the increments measured above, the distance between the first and second fluorescent bands was measured as dentin apposition over 7 days. We determined that the average weekly growth rate was $68.5 \pm 3.3 \mu \mathrm{m}$, and the periodicity of the incremental growth lines was $8.23 \pm 0.25 \mathrm{hr}$ (Table 1).

The 8-hr increments were sustained in wild-type and Bmal1deficient mice housed under constant darkness

Next, we analyzed both types of rhythmic patterns of molar dentin in wild-type mice housed in $\mathrm{DD}$, to examine whether the periodic increments are sustained without external time-giving cues because light is a primary zeitgeber. The average intervals of incremental growth lines and fluorescent bands were $3.26 \pm 0.27$ and $68.1 \pm 2.4 \mu \mathrm{m}$, respectively, and the periodicity of incremental growth lines was calculated to be $8.03 \pm 0.43 \mathrm{hr}$, similar to the computed periodicity in the LD cycle (Fig. 2A, B; Table 1). These results indicated that the 8-hr increments in mouse molar dentin were sustained in the free-running environment. Moreover, we examined the rhythmic patterns using $\mathrm{Bmal1}^{-1-}$ mice housed in DD and observed incremental growth lines at a spacing of $3.04 \pm 0.21 \mu \mathrm{m}$ (Fig. 2C; Table 1). The distance between neighboring labels was $61.9 \pm 5.5$ $\mu \mathrm{m}$; therefore, the incremental growth lines were estimated to be produced in $8.27 \pm 0.38 \mathrm{hr}$ (Fig. 2D; Table 1). These findings demonstrated that the approximately 8 -hr increments were also sustained in $\mathrm{Bmall}^{-/-}$mice under conditions of constant darkness.

\section{Dentinogensis in mouse molars was complete before the mouse reach 4 weeks of age}

Since the number of fluorescent bands did not match the frequencies of tetracycline administrations, we performed additional experiments where the mice were administrated with tetracycline once per week for four weeks. Three fluorescent bands were observed in the coronal dentin when the tetracycline was injected weekly at 1 4 weeks of age (Fig. 3A). On the other hand, weekly administrations of tetracycline at $5 \sim 8$ weeks of age produced no tetracycline-labeled bands in the coronal dentin (Fig. 3B). These results indicated that dentinogenesis in mouse molars is complete before the mouse reaches 4 weeks of age.

\section{Discussion}

For the first time, in the present study, we demonstrated that incremental growth lines in mouse molar dentin were generated at approximately 8 -hr intervals, using tetracycline as the chronological growth marker. The 8-hr increments were sustained under constant darkness, suggesting that rhythmic dentinogenesis in mouse molar dentin underlies the self-sustained rhythms. Further, the ultradian dentin increments were observed in $\mathrm{Bmall}^{-/-}$mice housed in DD. BMAL1 is an essential transcription factor for circadian transcription, and the $\mathrm{Bmal1}^{-/}$mouse becomes behaviorally circadian arrhythmic in constant darkness [3]. Our results demonstrated that the 8-hr ultradian increments in mouse molar dentin are regulated independently from the circadian clock. This was also supported by previous reports, which suggested that ultradian locomotor activity oscillations persisted in rodents housed in DD, even upon ablation of SCN [8] or genetic disruption of the circadian clocks [3]. An 8-hr ultradian rhythm was also observed in circulating endothelin-1 (ET-1) in human blood [6], and a study using dental pulp stem cells suggested that ET-1 promotes odontogenic differentiation [12], suggesting that 

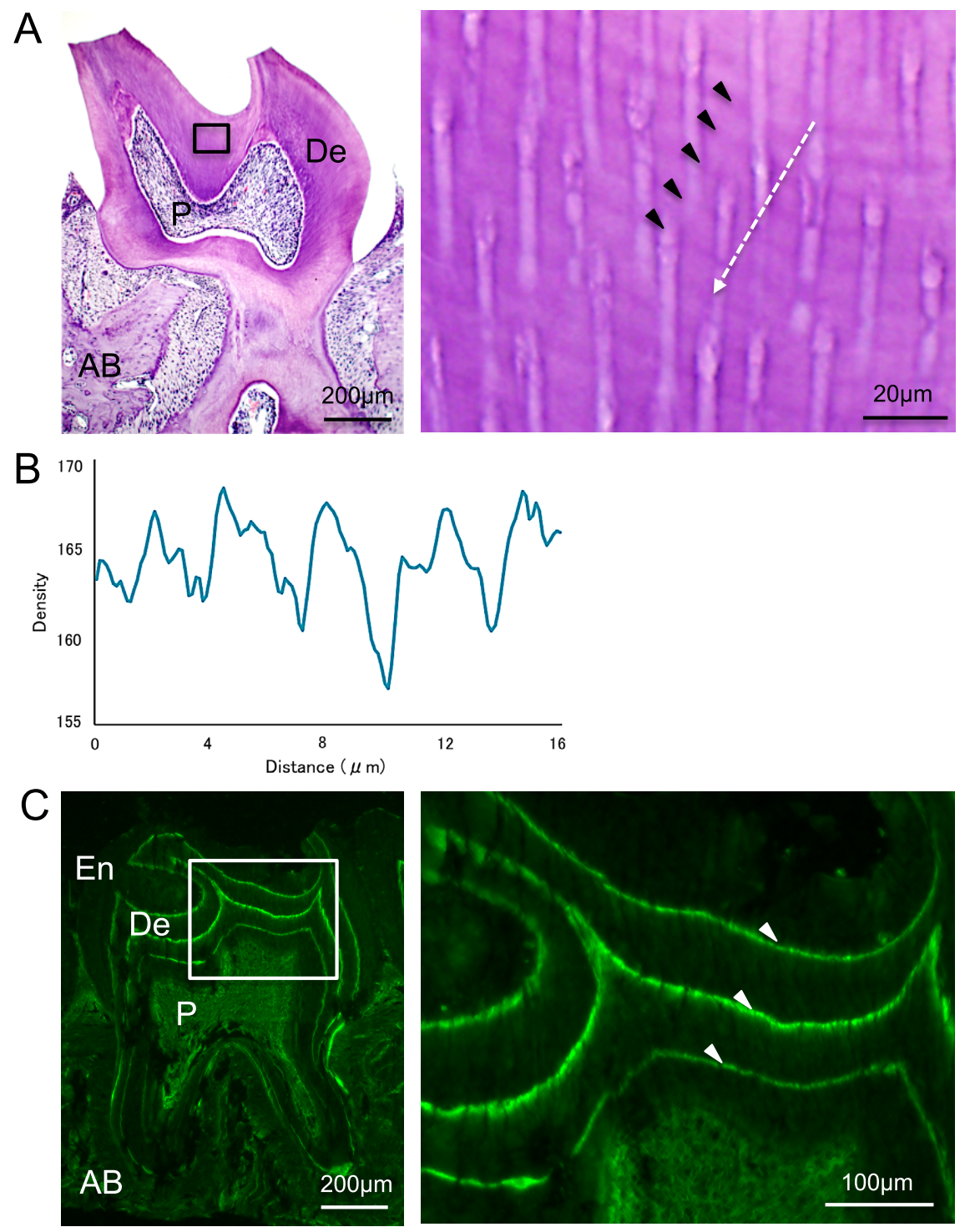

Fig. 1. Representative microscopic images of upper second molars from the wild-type mice in the 12:12 hr LD cycle. Enlarged views of the region marked in the square are shown on the right. A: Decalcified section of the molar stained with hematoxylin and eosin. Incremental growth lines are indicated with arrowheads. The white arrow indicates the direction measured intensity in panel (B). Enamel is lost during decalcification. B: Densitometric pattern obtained by scanning the increments shown in panel (A). The $\mathrm{x}$-axis represents a distance along the arrow, and the y-axis is the pixel intensity. C: Fluorescence microscopic image of frozen sections. Tetracycline-labeled bands are indicated with arrowheads. Enamel is retained at the outer side of the dentin. De: dentin; P: pulp; AB: alveolar bone, En: enamel. Bars $=200 \mu \mathrm{m}$ (A \& C, left); $20 \mu \mathrm{m}(\mathbf{A}$, right); $100 \mu \mathrm{m}(\mathbf{C}$, right).

Table 1. Averaged intervals of incremental growth lines (IL) and tetracycline-labeled bands (TB), and the periodicity of incremental growth lines

\begin{tabular}{|c|c|c|c|}
\hline Samples & $\mathrm{IL}(\mu \mathrm{m})$ & $\mathrm{TB}(\mu \mathrm{m})$ & Periodicity (hr) \\
\hline WT in LD $(n=5)$ & $3.36 \pm 0.09$ & $68.5 \pm 3.3$ & $8.23 \pm 0.25$ \\
\hline WT in DD $(n=4)$ & $3.26 \pm 0.27$ & $68.1 \pm 2.4$ & $8.03 \pm 0.43$ \\
\hline Bmal1 $^{-1-}$ in DD $(\mathrm{n}=4)$ & $3.04 \pm 0.21$ & $61.9 \pm 5.5$ & $8.27 \pm 0.38$ \\
\hline ANOVA $\quad P$ value & $0.092(\mathrm{NS})$ & $0.060(\mathrm{NS})$ & $0.581(\mathrm{NS})$ \\
\hline$F$ value & 4.103 & 4.103 & 4.103 \\
\hline
\end{tabular}

n: number of animals. Data are presented as mean \pm SD. NS: not significant. 

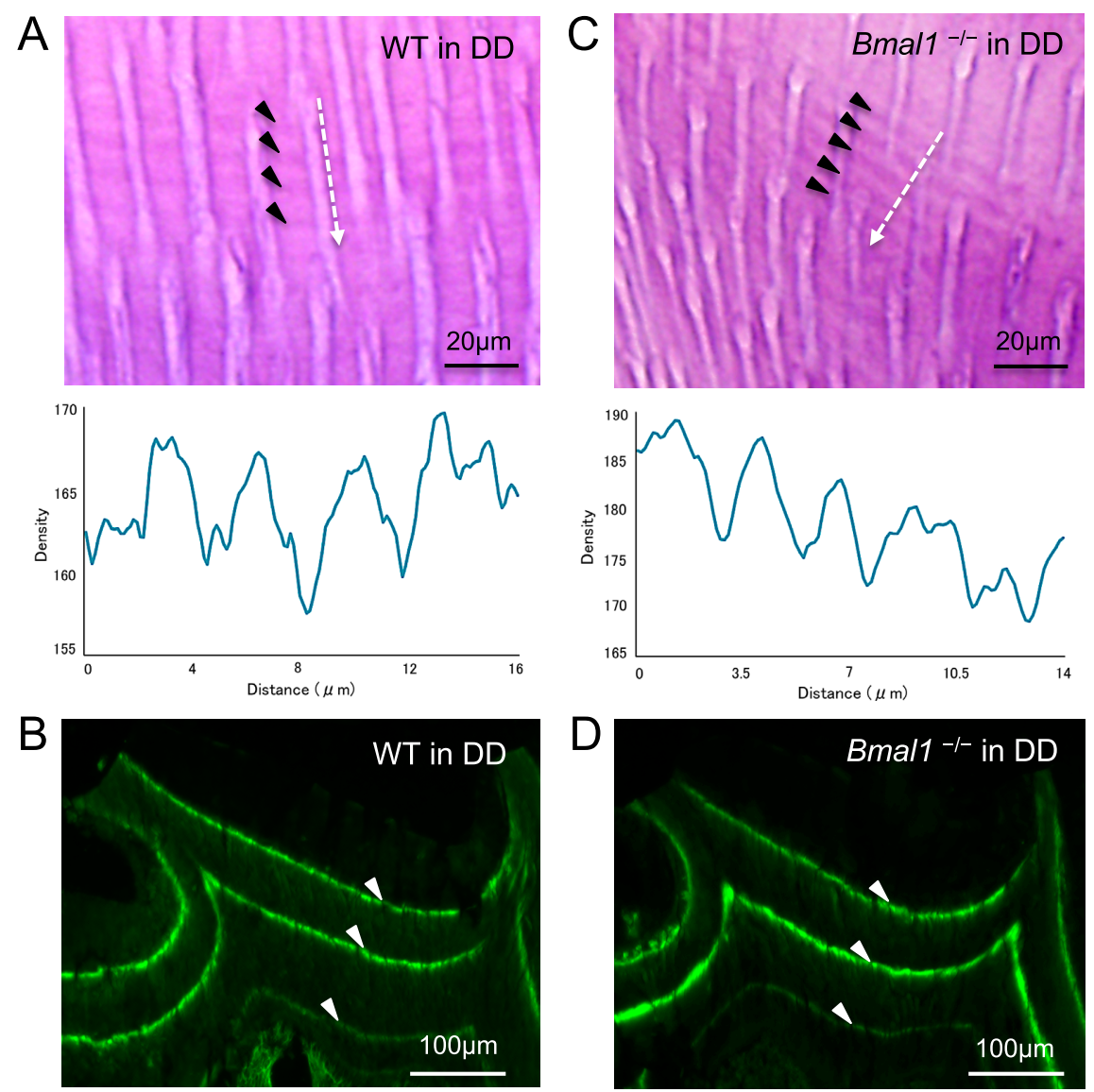

Fig. 2. Typical light and fluorescent microscopic observations of upper second molars from the wild-type and Bmal1 ${ }^{-/-}$mice in DD. Decalcified sections and densitometric patterns from wild-type (A) and Bmal1 $^{-/-}$(C). Frozen sections from wild-type (B) and Bmall ${ }^{-/-}(\mathbf{D})$. Bars $=20 \mu \mathrm{m}(\mathbf{A}, \mathbf{C}) ; 100 \mu \mathrm{m}(\mathbf{B}$, D).
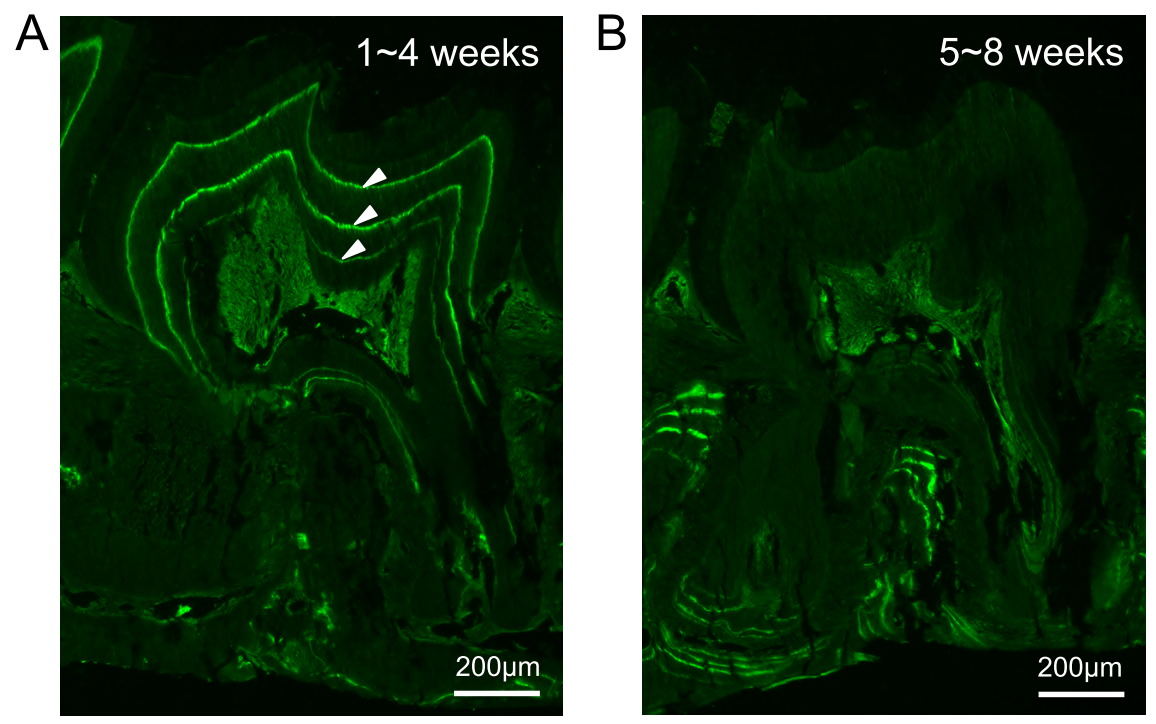

Fig. 3. Representative fluorescent microscopic images from mice housed in LD and administrated with tetracycline each week (for 4 weeks) at 1 4 (A) or 5 8 (B) weeks of age. Three fluorescent bands in crown dentin can be observed in panel (A), and no labels can be observed in panel (B), respectively. Bars $=200 \mu \mathrm{m}$. 
ET-1 may contribute to generation of the 8-hr increments. However, the molecular mechanisms controlling the 8-hr ultradian rhythms are widely unknown. Further research is warranted to elucidate the mechanism by which the rhythmic increments are generated.

Since the 1930s, incremental growth lines in incisor dentin of rodents reportedly showed a circadian pattern [18, $23,24]$. Rodent incisors were often selected as research targets because they provide a unique and powerful model for studying stem cells of the epithelium and mesenchyme, due to continuous incisor growth throughout the rodent's lifespan [14]. In contrast, rodent molars exhibit limited growth and stop growing after crown formation $[4,13]$. The circadian clock genes (Bmall, Clock, Perl and Per2) are reportedly expressed in mouse molars and are involved in dentin matrix secretion and mineralization [35]. However, our results demonstrated that the mouse molar dentin exhibited 8-hr ultradian increments, suggesting that the rhythmicity of dentinogenesis differs between incisors and molars. The question whether this difference is related to continuous or non-continuous tooth growth is of considerable interest. In our experiments, we measured dentin growth rate as intervals of the first and second tetracycline-labeled bands from DEJ in the coronal dentin. Dentinogenesis in the mouse second molar reportedly started from postnatal day 2 (P2) and attained complete functional occlusion at P25 [4], which support our findings that the incremental growth lines were formed at 1 2 weeks of age. Circadian rhythms at the individual cellular level are formed in the late embryonic stage [27], whereas overt circadian rhythms in the tissue or individual levels occur after birth [33]. The basal corticosterone level undergoes daily changes from P22 in rats [11] and an activity rhythm is found by P9 [25]. These findings suggest that dentinogenesis in mouse molars stops before the emergence of circadian physiological rhythms at the whole body levels. This is supported by a study using rat incisors in which ultradian dentin increments were observed at 1 week of age and circadian components appeared later [18]. We could not eliminate the possibility that there are functional differences in odontoblasts between mouse molars and incisors, which produce rhythmic increments with a different periodicity. However, our results clearly demonstrated that the dentinogenesis in mouse molar underlie the 8-hr ultradian rhythms and that the circadian clock protein, BMAL1, is not required for the ultradian increments. These results provide new insight into tooth growth. Future studies are warranted to understand the biological mechanisms controlling rhythmic tooth growth and its functional significance.

\section{Conflicts of Interest}

The authors declare that there are no conflicts of interest.

\section{Acknowledgments}

We would like to thank the members of the Department of Physiology and Systems Bioscience, Kyoto Prefectural University of Medicine for their valuable discussions and technical support. This work was supported in part by grants-in-aid for scientific research from the Japan Society for the Promotion of Science to K.Y and the Cooperative Research Program of Primate Research Institute of Kyoto University to R.O (2018-B-21, 2019-B-19).

\section{References}

1. Andresen, V. (1898) Die querstreifung des dentins. "Deutsche monatsschrift fur zahnheilkunde", pp. 386-389.

2. Balsalobre, A., Damiola, F. and Schibler, U. (1998) A serum shock induces circadian gene expression in mammalian tissue culture cells. Cell 93; 929-937.

3. Bunger, M. K., Wilsbacher, L. D., Moran, S. M., Clendenin, C., Radcliffe, L. A., Hogenesch, J. B., Simon, M. C., Takahashi, J. S. and Bradfield, C. A. (2000) Mop3 is an essential component of the master circadian pacemaker in mammals. Cell 103; 10091017.

4. Cohn, S. A. (1957) Development of the molar teeth in the albino mouse. Am. J. Anat. 101; 295-319.

5. Dean, M. C. (1998) Comparative observations on the spacing of short-period (von Ebner's) lines in dentine. Arch. Oral Biol. 43; $1009-1021$.

6. Herold, M., Cornelissen, G., Loeckinger, A., Koeberle, D., Koenig, P. and Halberg, F. (1998) About 8-hour variation of circulating human endothelin-1. Peptides 19; 821-825.

7. Hosokawa, T., Tsuchiya, Y., Okubo, N., Kunimoto, T., Minami, Y., Fujiwara, H., Umemura, Y., Koike, N., Kubo, T. and Yagita, K. (2015) Robust Circadian Rhythm and Parathyroid HormoneInduced Resetting during Hypertrophic Differentiation in ATDC5 Chondroprogenitor Cells. Acta Histochem. Cytochem. 48; $165-171$.

8. Ibuka, N., Inouye, S. I. and Kawamura, H. (1977) Analysis of sleep-wakefulness rhythms in male rats after suprachiasmatic nucleus lesions and ocular enucleation. Brain Res. 122; 33-47.

9. Kawamoto, T. and Shimizu, M. (2000) A method for preparing 2- to 50- $\mu \mathrm{m}$-thick fresh-frozen sections of large samples and undecalcified hard tissues. Histochem. Cell Biol. 113; 331-339.

10. Kawashima, N. and Okiji, T. (2016) Odontoblasts: Specialized hard-tissue-forming cells in the dentin-pulp complex. Congenit. Anom. (Kyoto). 56; 144-153.

11. Levin, R. and Levine, S. (1975) Development of circadian periodicity in base and stress levels of corticosterone. Am. J. Physiol. 229; 1397-1399.

12. Liu, M., Zhao, L., Hu, J., Wang, L., Li, N., Wu, D., Shi, X., Yuan, M., Hu, W. and Wang, X. (2018) Endothelial cells and endothelin1 promote the odontogenic differentiation of dental pulp stem cells. Mol. Med. Rep. 18; 893-901.

13. Lungova, V., Radlanski, R. J., Tucker, A. S., Renz, H., Misek, I. and Matalova, E. (2011) Tooth-bone morphogenesis during postnatal stages of mouse first molar development. J. Anat. 218; 699-716.

14. Mao, J. J. and Prockop, D. J. (2012) Stem cells in the face: tooth regeneration and beyond. Cell Stem Cell 11;291-301.

15. McGuigan, H. and Brough, G. A. (1923) RHYTHMIC BANDING OF PRECIPITATES (LIESEGANG'S RINGS). $J$. Biol. Chem. 58; 415-423.

16. Mishima, H. (2003) The short-period incremental lines in dentin 
of Alligatoridae Teeth. In "Biomineralization (BIOM 2001): formation, diversity, evolution and application", pp. 317-320.

17. Ohno, K. (1978) On the factors influence the pattern of tetracycline labeling of developing hard tissues. Japanese Journal of Oral Biology 20; 252-269.

18. Ohtsuka, M. and Shinoda, H. (1995) Ontogeny of circadian dentinogenesis in the rat incisor. Arch. Oral Biol. 40; 481-485.

19. Ohtsuka, M., Saeki, S., Igarashi, K. and Shinoda, H. (1998) Circadian rhythms in the incorporation and secretion of $3 \mathrm{H}-$ proline by odontoblasts in relation to incremental lines in rat dentin. J. Dent. Res. 77; 1889-1895.

20. Ohtsuka-Isoya, M., Hayashi, H. and Shinoda, H. (2001) Effect of suprachiasmatic nucleus lesion on circadian dentin increment in rats. Am. J. Physiol. Regul. Integr. Comp. Physiol. 280; R13641370.

21. Okada, M. (1943) Hard tissues of animal body-highly interesting details of Nippon studies in periodic patterns of hard tissues are described. Shanghai Evening Post Special Medical Edition of Sept.; 26-31.

22. Owen, R. (1840) Odontography; Or, A Treatise on the Comparative Anatomy of the Teeth; Their Physiological Relations, Mode of Development, and Microscopic Structure, in the Vertebrate Animals. vol. v. 1, H. Baillière, London.

23. Schour, I. and Hoffman, M. M. (1939) Studies in Tooth Development: II. The Rate of Apposition in Enamel and Dentin in Man and Other Mammals. J. Dent. Res. 18; 161-175.

24. Shinoda, H. and Ogura, H. (1977) A study on the circadian rhythms of dentine formation in rodents. Japanese Journal of Oral Biology 19; 481-489.

25. Smith, G. K. and Anderson, V. (1984) Effects of maternal isolation on the development of activity rhythms in infant rats. Physiol. Behav. 33; 751-756.

26. Takahashi, J. S. (2017) Transcriptional architecture of the mammalian circadian clock. Nat. Rev. Genet. 18; 164-179.

27. Umemura, Y., Koike, N., Ohashi, M., Tsuchiya, Y., Meng, Q. J., Minami, Y., Hara, M., Hisatomi, M. and Yagita, K. (2017) Involvement of posttranscriptional regulation of Clock in the emergence of circadian clock oscillation during mouse development. Proc. Natl. Acad. Sci. U S A 114; E7479-E7488.

28. Von Ebner, V. R. (1906) Uber die Entwicklung der leimgebenden Fibrillen im Zahnbein (Development of collagenous fibrils in the dentin). Sitzgsber. Akad. Wiss. Wien, Math.-naturwiss. Kl. 3; 281-346.

29. Weinreb, M. M., Assif, D. and Michaeli, Y. (1967) Role of attrition in the physology of the rat incisor. I. The relative value of different components of attrition and their effect on eruption. J. Dent. Res. 46; 527-531.

30. Xu, H., Snider, T. N., Wimer, H. F., Yamada, S. S., Yang, T., Holmbeck, K. and Foster, B. L. (2016) Multiple essential MT1MMP functions in tooth root formation, dentinogenesis, and tooth eruption. Matrix Biol. 52-54; 266-283.

31. Yagita, K., Tamanini, F., van Der Horst, G. T. and Okamura, H. (2001) Molecular mechanisms of the biological clock in cultured fibroblasts. Science 292; 278-281.

32. Yamazaki, S., Numano, R., Abe, M., Hida, A., Takahashi, R., Ueda, M., Block, G. D., Sakaki, Y., Menaker, M. and Tei, H. (2000) Resetting central and peripheral circadian oscillators in transgenic rats. Science 288; 682-685.

33. Yamazaki, S., Yoshikawa, T., Biscoe, E. W., Numano, R., Gallaspy, L. M., Soulsby, S., Papadimas, E., Pezuk, P., Doyle, S. E., Tei, H., Sakaki, Y., Block, G. D. and Menaker, M. (2009) Ontogeny of circadian organization in the rat. J. Biol. Rhythms 24; 55-63.

34. Yoo, S. H., Yamazaki, S., Lowrey, P. L., Shimomura, K., Ko, C. H., Buhr, E. D., Siepka, S. M., Hong, H. K., Oh, W. J., Yoo, O. J., Menaker, M. and Takahashi, J. S. (2004) PERIOD2::LUCIFERASE real-time reporting of circadian dynamics reveals persistent circadian oscillations in mouse peripheral tissues. Proc. Natl. Acad. Sci. U S A 101; 5339-5346.

35. Zheng, L., Papagerakis, S., Schnell, S. D., Hoogerwerf, W. A. and Papagerakis, P. (2011) Expression of clock proteins in developing tooth. Gene Expr. Patterns 11; 202-206.

This is an open access article distributed under the Creative Commons Attribution License, which permits unrestricted use, distribution, and reproduction in any medium, provided the original work is properly cited. 